\title{
Smooth Handover and Optimized Uplink Routing in Cellular IPv6 Networks
}

\author{
Rola Naja and Samir Tohmé \\ Ecole Nationale Supérieure des Télécommunications \\ 46, Barrault Street, 75634 Paris \\ \{rola.naja, samir.tohme\}@enst.fr
}

\begin{abstract}
Cellular IPv6 is an efficient IP micro-mobility protocol that provides important features such as easy global migration, cheap passive connectivity, efficient location management and routing, and flexible handover. However, Cellular IPv6 presents some problems when dealing with intra-domain traffic. In this paper, we propose an enhanced uplink routing mechanism coupled with a study of a smooth handover for intra-domain traffic. Simulation is carried out in order to show the performance results.
\end{abstract}

Keywords: Micro-mobility, Macro-mobility, Cellular IP, Handover.

\section{Introduction}

Provision of real time multimedia services to mobile users is the main objective of the next generation wireless networks, which will be IP-based and are expected to inter-work with the Internet backbone seamlessly. Two major challenges exist in wireless mobile networks, namely the fast handoff and the provision of Quality of Service (QoS) over IP-based wireless access networks.

Research efforts are oriented towards the design of IP micro-mobility protocols that can handle the IP mobility seamlessly. A suitable IP mobility protocol must resolve the following contradiction: it must keep the mobile's IP address unchanged in order to identify an end-system having a TCP session and at the same time it must deal with an IP address that changes with the mobile location. The Mobile IP architecture 2] was conceived in order to resolve the above-mentioned contradiction by using two IP addresses for a mobile node:

- The Home Address: is a permanent address used to uniquely identify the mobile node on the Internet.

- The Care-of Address (CoA): is a temporary address used to route the datagrams destined to the mobile node to the current point of attachment of the mobile.

As a result, Mobile IP allows the mobile nodes to change their point of attachment in an IP network. Unfortunately, Mobile IP has some limitations when applied to wide-area wireless networks with high mobility users that may require 


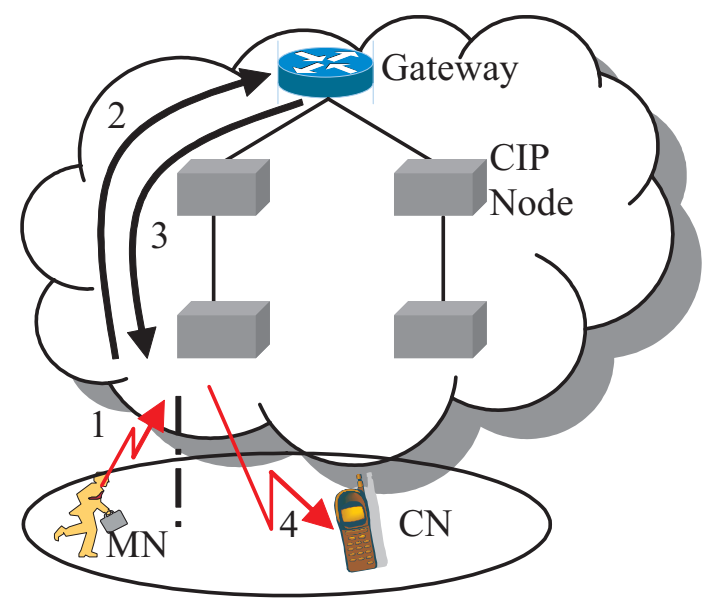

Fig. 1. Non optimization of Uplink Routing Mechanism for Intra-network traffic in Cellular IPv6 Network

quality of service. Thus, Mobile IP needs to be enhanced to cope with micromobility, i.e., movement across multiple subnetworks with frequent handovers and within a single network or domain.

IP Micro-mobility protocols were introduced in order to limit the disruption to user traffic during handoff: they complement Mobile IP by providing fast and also seamless handoff control. One promising micro-mobility protocol is Cellular IPv6 (CIP). Cellular IPv6 inherits cellular technology principles for mobility management, passive connectivity and handoff support, but implements these around the IP paradigm. As defined in [3], Cellular IPv6 suffers from a "nonoptimization" of the uplink routing in the case of intra-network traffic. In fact, all the traffic coming from the mobile node (MN) must pass through the gateway before being delivered to the corresponding node $(\mathrm{CN})$, even if the MN and the $\mathrm{CN}$ are connected to the same base station. Far from an optimal path, this kind of routing increases unduly the delay and the jitter of the packets. It can also imply the waste of bandwidth which constitutes a problem in case of high traffic (figure 1).

D. Gatzounas et al. [9] proposed an uplink routing optimization for Cellular IP networks that improves the protocol performance. In their proposition, even if the corresponding node and the mobile node are not in the same Cellular IP domain, all the Route caches in the route towards the gateway will be checked in order to find if $\mathrm{CN}$ has an entry with a "Tear down optimized flag" unset. This would cause an unnecessary delay for the cache processing. On the other hand, the overall performance is made at the expense of an increase of the signaling load.

In our study, we achieve two objectives. First, we enhance the uplink routing mechanism by adopting the mechanism proposed in [9] and by improving it in 
order to have faster lookup, better scalability and less frequent signaling messages. Second, we propose to study the performance of a smooth and anticipated handover in the Cellular IPv6 network. More precisely, we evaluate the performance of a buffering mechanism while taking into consideration the optimized uplink routing mechanism.

To this end, we start our study by briefly exposing the protocol Cellular IPv6 in section 2. Our proposition is introduced in section 3 discussing the extensions made to the Cellular IPv6 protocol. A simulation exposed in section 4 finalizes the study before concluding the paper.

\section{Cellular IPv6 Protocol}

The Cellular IPv6 proposal 3] uses specialized domain routers with host-based entries for local mobility and Mobile IP for inter-domain mobility. Thus, updates can be localized, enhancing the scalability of update mechanisms and limiting disruption.

Cellular IPv6 operates on mobile hosts, base stations and Internet gateways. It combines the cellular network with the IP network. Through paging and other handoff techniques, low-latency handoff and efficient management can be achieved.

\subsection{Cellular IPv6 Routing}

In Cellular IPv6, routing is based on host routes for the mobile host, through all the nodes on the path of the mobile host to the gateway. Cellular IPv6 provides two parallel cache systems that store the host-based routing entries. These caches are the Route cache and the Paging cache.

Each Cellular IPv6 node has an uplink neighbor and a downlink neighbor. To minimize control messaging, regular data packets transmitted by mobile hosts are used to refresh host-based routing entries stored in the caches. Cellular IPv6 nodes monitor mobile originated packets and maintain a distributed, hop-by-hop location database that is used to route packets to mobile hosts.

\subsection{Cellular IPv6 Handover}

Handoff is required when there is a change of access point during active transmission. Cellular IPv6 supports two types of handoff schemes. Cellular IPv6 hard handoff uses an algorithm that trades off some packet loss in exchange for minimizing handoff signaling. Cellular IPv6 semi-soft handoff tries to proactively notify the new access point before actual handoff. Semi-soft handoff minimizes packet loss and provides improved TCP and UDP performance over hard handoff. For the wireless technologies that do not provide the simultaneous connections, the indirect semi-soft handoff is proposed. 


\section{Proposition and Enhancements}

The Cellular IPv6 protocol uses two parallel cache systems in order to have faster lookup and better scalability. In fact, since only a portion of the hosts will be in active state at any given time, it is better to separate the caches for active and idle mobile hosts. Following the same reasoning, we propose to apply two parallel uplink routing mechanisms, namely the non-optimized uplink routing mechanism specified in [3] and the optimized uplink routing mechanism that we will detail in subsection 3.2 .

When the MN wants to send packets, it checks the subnet prefix of the CN's address.

- If the IPv6 subnet prefix of the MN's CoA is equal to the IPv6 subnet prefix of the CN's CoA, then the MN sets the flag OR, as defined in section 3.1 to 1 . As a result, the CIP nodes will apply the optimized uplink routing mechanism.

- Otherwise, the MN sends the packets without setting the flag OR. Then, the CIP nodes will route the packets with the non-optimized uplink routing mechanism.

This routing mechanism distinction will prevent the CIP nodes from scanning the caches mappings at each packet reception. Consequently, this will decrease the processing load in the nodes.

The optimized uplink routing proposed in [9] was made at the expense of an increase of the signaling load. In fact, in order to retain the routing cache consistency, the optimizing node must send a "proxy route-update" packet at a rate just faster than the route-timeout 11. In our mechanism, we propose to reduce this signaling load.

The loss of packets during the transition between networks should be minimal. It is shown in some research studies that buffering packets improves the global performance of Mobile IP. This paper defines a buffering mechanism that attempts to meet this goal for Cellular IPv6.

\subsection{Protocol Extensions}

Before detailing the optimized uplink routing mechanism, let us distinguish between the two following types of CIP nodes (figure 2):

1. Optimizing Node. This node is the intersection of the path from the MN's access node to the gateway router with the path from the CN's access node to the gateway router. Only one optimizing node at any time performs route optimization for a single pair of communicating mobile nodes in the same CIP network. The optimizing node must route data through the optimal path to the destination address.

${ }^{1}$ The route-timeout is the validity time of mappings in the Route caches. 


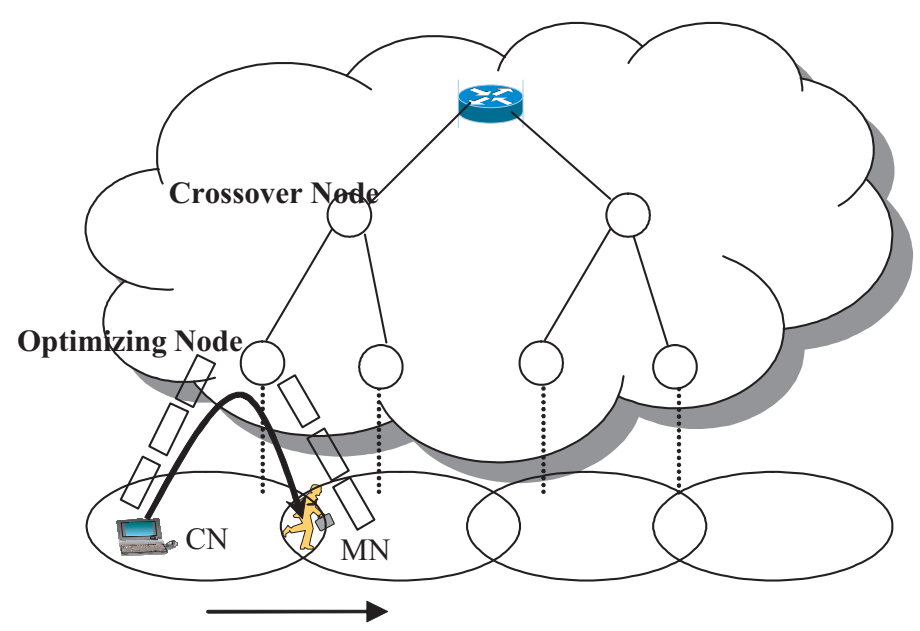

Fig. 2. Optimizing and Crossover Nodes in Cellular IPv6 Networks

2. Crossover Node. This node is the intersection of the old path from the gateway to the previous access node (base station) with the path from the gateway to the new access node (base station). The crossover node has two mappings for the mobile during the handover.

Let us define the following messages and flags added to the Cellular IPv6 protocol:

1. Proxy route-update message: it is sent by the crossover node towards the gateway [9. It is an IPv6 packet carrying a Hop-by-Hop Options extension header.

2. Remove Mapping message: it is sent by a mobile upon handoff. It is an IPv6 packet carrying a Hop-by-Hop Options extension header.

3. Optimize Route (OR) flag : it is carried in the Hop-by-Hop Options extension header of the data packets. When set, the route optimization must be performed by the nodes receiving the packets (default $=0$ ).

4. Duplicate (DUP) flag: it is in the IP header of the data packets indicating, when set, that the current packets are duplicated (default $=0$ ).

5. Optimize (ON) flag: it is in the Route caches' mappings (default=3).

If a node has, in its Route cache, a mapping for a mobile node $\mathrm{MN}$ with $\mathrm{ON}$ equal to:

- 0 , then the node "prepares" itself to be an optimizing node for the mobile node and its corresponding node. The $\mathrm{ON}$ is set to 0 during the handover establishment. The node in question does not send data through the optimal path as long as $\mathrm{ON}$ is equal to 0 , in order to prevent the packets routing to the new cell during handover.

-1 , then the node can be an optimizing node for the MN and its corresponding mobile node. The optimizing node must send data through the optimal path. 


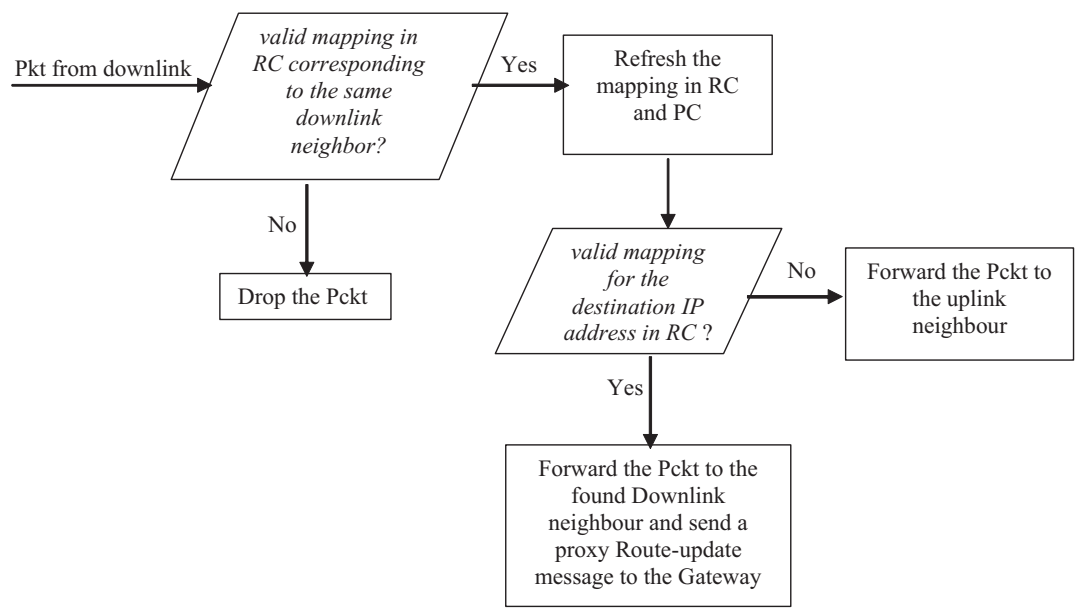

Fig. 3. Optimized Uplink Routing Mechanism in Cellular IPv6 Network (RC and PC are the Route and Paging caches)

- 2 , then the data packets sent by the mobile node MN must be duplicated by the node if it is an optimizing node.

\subsection{Optimized Uplink Routing Mechanism}

When a data packet arrives to a Cellular IPv6 node, the following events occur (figure 3):

If the data packet arrives from a downlink neighbor, the Route cache entry of the source IP address is searched first in the current node's Route cache. If the data packet arrives from a different neighbor than the one that is in its mapping or no mapping exists for the IP address, then the packet is dropped.

Otherwise, if the data packet is coming from the same neighbor, the mapping is refreshed in the Route and Paging caches.

The current node checks if the destination IP address has a valid mapping in the Route cache. There are two cases:

1. If such a mapping exists, the packet is forwarded to the downlink neighbor found in the mapping. In this case, the current node becomes an optimizing node for the two communicating mobile nodes.

2. Otherwise, if the Route Cache contains no mapping for the destination IP address then the packet is forwarded to the uplink neighbor.

In the first case, the optimizing node generates a proxy route-update message towards the gateway. The proxy route-update contains the IP address of the MN in the control field of the Hop-by-Hop Options extension header. The sending rate of the proxy route-update in [9] is controlled by a time interval shorter than 
the route-timeout interval $(9 \mathrm{~s})$. Thus, more signaling load is generated than in the non-optimized uplink routing. We propose that the optimizing node sends the proxy route-update message once: this message is sent when the node receives the first data packet that causes the node to become an optimizing node.

The proxy route-update sets the ON, in the mapping corresponding to the $\mathrm{MN}$, to 1 . If it finds ON equal to 2, no change in the mapping will be done.

Moreover, the proxy route-update will set the expiration time in the MN's mapping to the sum of the current time and an estimated time. The latter will be estimated according to the dwell time of the mobile in the CIP domain (according to the speed of the mobile) and to the call duration (according to the traffic type). In this case, the CIP nodes will operate in a hard state. This will help to reduce the signaling load and will not affect the route cache consistency. With our mechanism, the caches will always be updated according to the mobiles' movement, and the packets will arrive to the correct destination to the mobiles movement. In fact, a change in the route cache occurs:

- When one of the two communicating mobiles leaves the CIP domain. If the sending mobile node leaves the cell, then it sends a "paging-tear down" 2 The latter removes the mappings corresponding to the sending mobile node in the caches. Whenever the receiver leaves the cell, then it also sends a paging-tear down. The latter removes the mappings corresponding to the receiver in the caches. The packets sent to the receiver, in this case, will pass through the gateway in the uplink direction. And these packets will refresh the caches.

- When the sending mobile node's traffic is forwarded uplink. In this case, the packets will refresh the caches of the optimizing node and will be sent to a new optimizing node.

- At the end of the session and after the expiration of an "idle time", the sending mobile node becomes idle. The mapping of the sending mobile node stored in the Route caches will expire. As a result, the optimizing node will be a regular Cellular IPv6 node. The sending mobile node sends frequent paging-update packets. These packets will refresh the Paging caches of all the nodes in the path leading to the gateway.

As a result, the caches are refreshed according to the mobility of the flows, to the session duration, ... Therefore, the proxy route-update can be sent less frequently without affecting the routing mechanism.

In order to retain the routing cache consistency, the optimizing node of a communicating hosts pair, when receiving the update messages coming from the sending mobile node, must refresh its caches. Nevertheless, it must prevent sending these messages upwards in order to prevent the refreshing of the caches belonging to the branch leading to the gateway. Otherwise, the expiration time in the caches' mappings will change from the estimated time value. In this case,

\footnotetext{
${ }^{2}$ A paging-teardown packet is an IPv6 packet with a Hop-by-Hop Options extension header where the source address is the IP address of the sending mobile node, the destination address is the Gateway and the Hop-by-Hop option is of Paging-teardown type 3 .
} 
this expiration time will be set to the sum of the current time and the Routeupdate time. This will cause the caches' entries to expire because the caches do not receive frequent proxy route-update messages.

\subsection{Handover Handling}

Not all wireless technologies have simultaneous connection capability, i.e. they cannot listen to the current BS while sending a route-update packet to the new BS. For this situation an indirect semi-soft handoff is used [3]. We propose to enhance the indirect semi-soft handover handling while taking into account the optimized uplink routing mechanism.

Our study defines a buffering mechanism for the indirect semi-soft handoff. This buffering mechanism reduces the loss and the packet delay during the handover. The key idea is that the optimizing node duplicates the packets destined to the moving receiver. The original packets will be routed via the optimal path. As for the duplicated packets, they are sent to the crossover node where they are stored. These duplicated packets will be routed to the new mobile location after handover. In this way, the delay and the packets loss will be optimized.

We assume that a call is set-up between a mobile node and a corresponding node. We suppose also that a mobile node can not send and receive packets at the same time. This assumption is made for the ease of the handover handling description.

When a mobile node performs handover, the following sequence of events occurs:

1. The mobile node sends a route-update packet to the current BS. This packet has the IP address of the new BS as destination IP address. The route-update packet contains the address of the corresponding mobile in the control field of Hop-by-Hop Options extension header. The I flag is set to indicate indirect semi-soft handoff.

We distinguish between two cases:

If the mobile node is a sending mobile node, then it sets the OR flag of the route-update packet to 1 .

If the mobile node is a receiver, then it sets the OR flag of the route-update packet to 0 . This implies that the packet will reach the gateway. In this way, the route-update packet will reach the crossover node. This will not happen when the optimized uplink routing mechanism is used $(\mathrm{OR}=1)$ and when the optimizing node is hierarchically under the crossover node.

The current BS forwards the route-update packet to the Gateway. The latter uses then normal IP routing to deliver the packet to the new BS. The route-update packet sets the flag $\mathrm{ON}$ to 2 in the Route caches mappings that correspond to the sending mobile node. This is done to all the nodes belonging to the branch starting from the current base station up to the gateway.

In the following steps, we consider that the mobile node that is performing handover is the receiver. This is an important case, since the packets sent to 
1: Route-update

2: Semi-soft route-update

3: Sending packets

4: Duplicating and sending packets

:........ Duplicated Packet

Original Packet

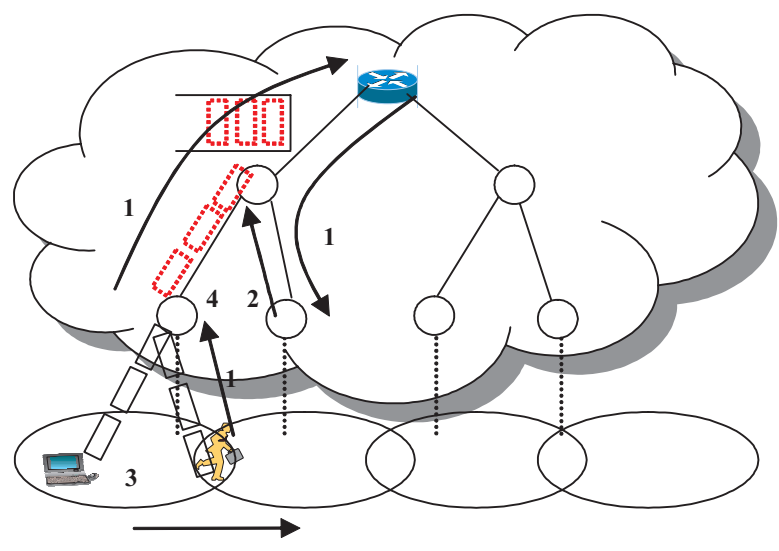

Fig. 4. Packets duplication during handover

1: Route-update

2: Paging-teardown

3: Sending duplicated packets

4: Remove-mapping

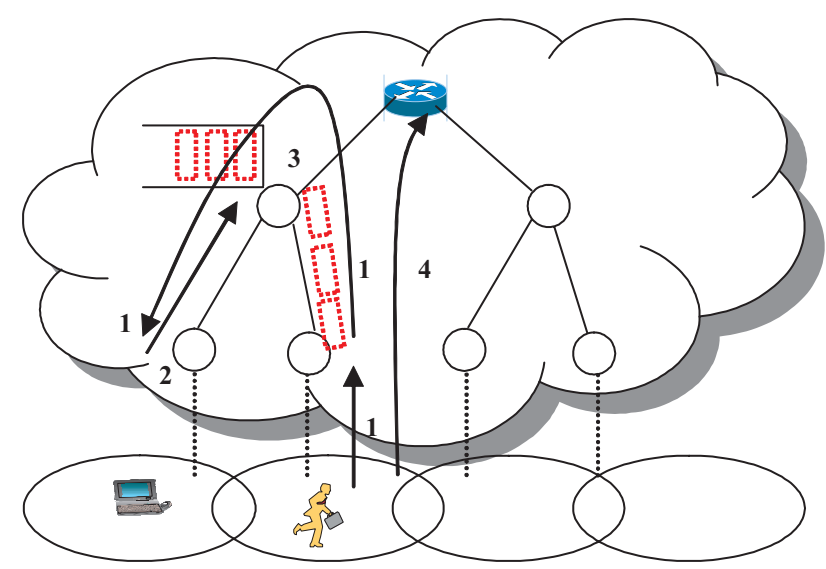

Fig. 5. Sending Packets after handover

the receiver must be duplicated in order to prevent the packets loss. When the mobile node that is performing handover is the sending mobile node, there is no need to perform packets duplication.

2. When the new BS receives the indirect semi-soft handoff packet, a semi-soft route update packet is created $(\mathrm{I}=0, \mathrm{~S}=1)$ with the IP address of the mobile host as the source address. It is then forwarded upstream. The semi-soft route-update packet creates new mappings in the Route and Paging Cache similarly to regular route-update packets. However, it sets the flag $\mathrm{ON}$ in the Route cache mapping that corresponds to the sending mobile node to 0 . This is done to prevent the routing of packets to the new cell before the handover takes place. Recall that we are proposing to study the indirect semi-soft handoff. 
When the semi-soft route-update packet reaches the crossover node where the old and new path meet, the new mapping is added to the cache instead of replacing the old one.

3. Packets that are sent to the mobile receiver must pass by the optimizing node. When the flag $\mathrm{ON}$, in the mapping corresponding to the sending mobile node, is equal to 2 , the optimizing node performs the duplication of the packets (figures 4). The original packets are routed using the optimizing routing mechanism. As for the duplicated packets, they are routed towards the crossover node and stored in the buffer located in the crossover node.

Consequently, even during handover, the packets are always sent via the optimal route, and the duplicated packets wait in the crossover node in order to be sent via the shortest path.

It is noteworthy that the duplicated packets do not refresh the caches in our proposition. Otherwise, the caches must be refreshed each route-update time: this would incur more signaling load. Thus, the flag DUP of the duplicated packets will be set to 1: the CIP nodes will be able to identify the duplicated packets.

4. When the mobile node moves to the new cell, it sends a route-update packet $(\mathrm{OR}=1, \mathrm{I}=\mathrm{S}=0)$ to the current $\mathrm{BS}$. This packet has as a destination IP address, the IP address of the new BS. The packet in question contains the address of the moving mobile and the corresponding node's address in the control field of the Hop-by-Hop Options extension header. The route-update packet sets $\mathrm{ON}$ to 3 in the Route cache mapping corresponding to the sending mobile node, if it finds ON equal to 2. The current BS will then forward this packet to the old base station.

5. When receiving the route-update packet, the old base station sends a pagingteardown packet $(\mathrm{OR}=0, \mathrm{I}=\mathrm{S}=0$ ) with the $\mathrm{IP}$ address of the receiver as in the source address. This packet contains the source address of the mobile and the corresponding mobile address in the control field of the Hop-by-Hop Options extension header. This paging-teardown removes all the mappings concerning the moving mobile in the Caches except for the ones pointing to the new Base Station.

When the paging-teardown arrives to the crossover node, it forces the buffer to free the packets sent to the receiver (figure [5). The freed packets take then the optimal path to arrive at the new mobile location.

6. The mobile node sends a remove-mapping message $(\mathrm{OR}=0, \mathrm{I}=\mathrm{S}=0)$ that contains the address of the corresponding mobile. This message will set $\mathrm{ON}$ in the mapping corresponding to the sending mobile node to 3 , if it finds it equal to 2. This is done in all the caches of the CIP nodes belonging to the branch leading to the gateway.

The handover is then complete.

\section{Simulation Results and Performance Analysis}

In order to study the performance of the proposed mechanism, simulations was carried out using OMNeT++ [11]. The CIP network illustrated in figure 2 was 


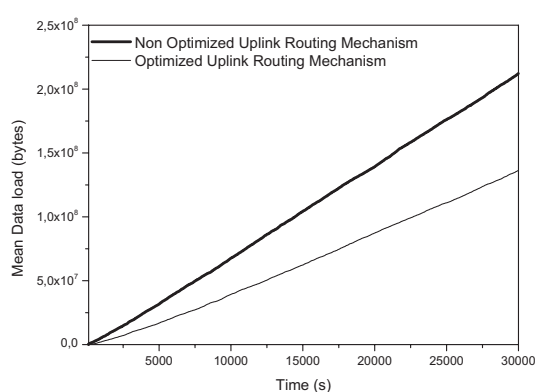

Fig. 6. Mean Data Load

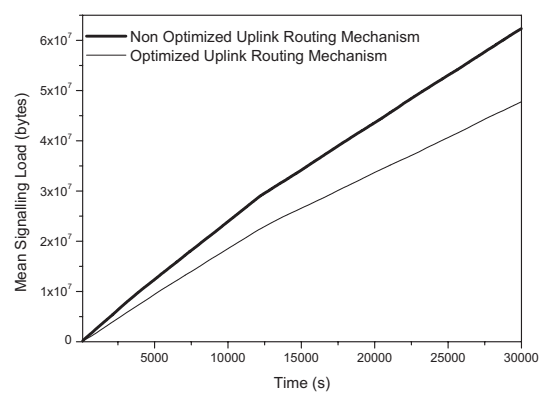

Fig. 7. Mean Signaling Load

simulated. We suppose that the wireless bandwidth is equal to $1 \mathrm{Mb} / \mathrm{s}$, the wired link capacity dedicated for data is $1,92 \mathrm{Mb} / \mathrm{s}$, that for the signaling is $128 \mathrm{~Kb} / \mathrm{s}$. The data traffic considered represents a typical WWW session $(64 K b / s)$ that consists of a sequence of packet calls. During a packet call, several packets may be generated. A time interval called reading time separates two consecutive packet calls. The parameters and laws that model the data traffic are specified in [10]. Mobile users are considered as pedestrians with mean speed of $1.8 \mathrm{Km} / \mathrm{h}$ moving within the cells of radius $0,1 \mathrm{Km}$. Moreover, we consider that each CIP node has 3 buffers:

- the first buffer is dedicated for the signaling packets Bs.

- the second buffer is allocated to the data packets Bd.

- the third buffer is for the duplicated data packets during the handover Bh.

Since the signaling packets are important in CIP network, the signaling buffer Bs is allocated a percentage of the link capacity. Thus, Bs does not suffer from the resource contention. One better alternative is to apply the Round-Robin mechanism between the signaling buffer and the data packets. This would improve the bandwidth use but it would not change our results nor the conclusions of our study.

We apply the Head Of the Line (HOL) discipline with no-preemption in order to schedule the packets in $\mathrm{Bd}$ and $\mathrm{Bh}$. The HOL serves the packets, stored in Bh first, after the paging-teardown reception. Note that the freed packets are the ones sent to the moving receiver which address is the source address of the paging-teardown.

\subsection{Numerical Results}

Figure [6] shows the data load measured on the gateway interfaces. One can see that the optimizing routing mechanism lowers the load on the gateway which is considered as a bottleneck in the CIP network. This result has also been obtained by [9]. However and unlike [9], the signaling load on the gateway interfaces is less than the one obtained with the non-optimized uplink routing 


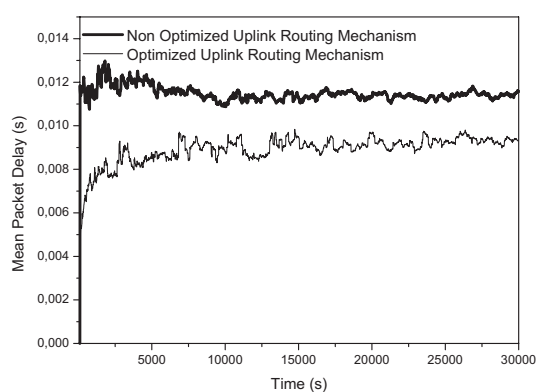

Fig. 8. Mean Data Packet Delay

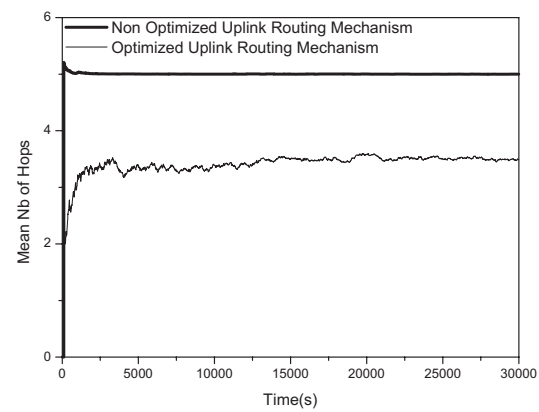

Fig. 9. Mean Number of Hops

mechanism (figure 7). In fact, the optimizing nodes retain all the update packets sent by the sending mobile node. This can alleviate the signaling load on the gateway. On the other hand, we argue that the proxy route-update packet must be sent once and that the expiration time, related to this packet, in the caches mappings must be well-chosen. Moreover, most of the signaling messages needed to establish the handover are sent through the optimal path. As a result, the decrease of the signaling load on the gateway interfaces is a logical consequence of our mechanism.

Figure 9 shows the mean number of hops crossed by the packets before arriving at destination. As we can see, this number of hops is constant with the non-optimized uplink routing mechanism. In fact, the packets always pass by the gateway before being routed to destination. Thus, the number of hops depend on the network topology. With the optimized uplink routing mechanism, the number of crossed hops is reduced. This is because the packets take the optimal path before arriving at destination.

As for the delay experienced by the packets, the curves depicted in figure 8 show better results than with the non-optimized uplink routing mechanism, due to the reduced number of hops crossed by the packets. This delay is also due to the duplicated packets that are received by the mobile upon sending the paging-teardown. As we can see, the localization of the storing buffer on the crossover node and not on the gateway helps to reduce the delay encountered by the packets.

Figure 10] depicts the delay of the packet sent during the handover. It can be seen that this delay is much higher with the non-optimized uplink routing mechanism. In fact, with our enhanced mechanism, the packets are sent through the optimal during and after handover.

We also measured the delay of establishment the handover in figure [1]. We found that this delay is higher with the non-optimized uplink routing mechanism. In fact, with the optimized uplink routing mechanism and when the mobile moves to the neighboring cell, the path taken by the route-update packet is shorter than that with the non optimized uplink routing mechanism. 


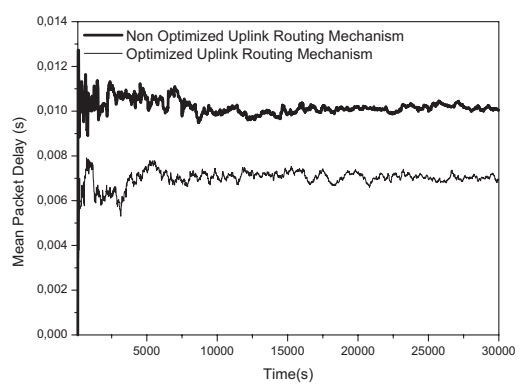

Fig. 10. Mean Data Packet Delay During Handover

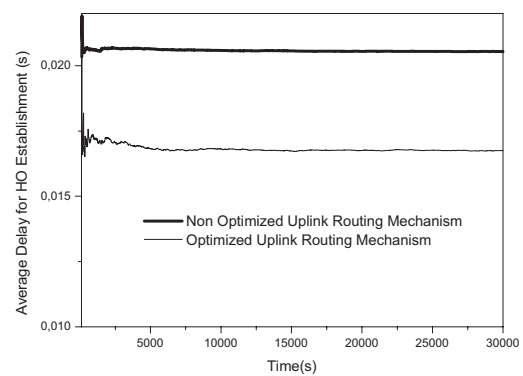

Fig. 11. Mean Handover Establishment Delay

The retrieved numerical results show the benefits of the enhanced optimized uplink routing mechanism. These better results were obtained at the expense of some complexity added to the CIP nodes. One must make the trade-off between better performance and complexity.

\section{Conclusion}

This paper presents an enhanced uplink routing mechanism coupled with a smooth handoff study. Our proposal aims to minimize the delay and the loss experienced by the data packets during communications and especially during handover. Simulation results show the good performance obtained at the expense of some complexity added to the Cellular IPv6 nodes.

Some open issues still remain. The resource allocation and the study of an efficient call admission control providing quality of service to different classes of mobile users are important issues to be addressed and need further investigation.

\section{References}

1. A.Campbell, J.Gomez, S.Kim, C.Wan, Z.Turanyi, A.Valko: Comparison of IP Micromobility Protocols. IEEE Wireless Commun. Mag., vol.9, no.1, February 2002.

2. D.B. Rice, C. Perkins: Mobility Support in IPv6. Internet draft, draft-ietf-mobileipipv6-15.txt, July 2001.

3. Z.D.Shelby, D.Gatzounas, A.Campbell, C.Wan: Cellular IPv6. Internet draft, draftshelby-seamoby-cellularipv6-00.txt, November 2000.

4. H. Soliman, C. Castelluccia, K. El-Malki, L.Bellevier: Hierarchical MIPv6 mobility management (HMIPv6). Internet draft, draft-ietf-mobileip-hmipv6-05.txt, July 2001.

5. R. Ramjee et al.: HAWAII: A Domain-Based Approach for Supporting Mobility in Wide-area Wireless Networks. Proc.IEEE Int'l.Conf.Network Protocols, 1999.

6. R. Koodli, C.Perkins: A framework for smooth handovers with Mobile Ipv6. Internet draft, draft-koodli-mobileip-smoothv6-00.txt, July 2000. 
7. K.EL-Malki, H.Soliman: Fast Handoffs in Mobile IPv4. Internet draft, draftelmalki-mobileip-fast-handoffs-03.txt, September 2000.

8. K. El-Malki, P.Calhoun, T. Hiller, J. Kempf, P.J.McCann, A.Singh, H.Soliman, S.Thalanany: Low Latency Handoffs in Mobile IPv4. Internet draft, draft-ietfmobileip-lowlatency-handoffs-v4-04.txt,July 2002.

9. P.Mähönen, D.Gatzounas, A. Inzerilli: Cellular IP Route Optimization. Internet draft, draft-shelby-cip-routeoptimization-00.txt, June 2001.

10. European Telecommunications Standards Institute ETSI, Universal Mobile Telecommunications Systems UMTS: Selection procedures for the choice of radio transmission technologies of the UMTS.TR101 112,V3.2.0,1998.

11. OMNeT++:Objective Modular Network Testbed in $\mathrm{C}++$. http://www.hit.bme.hu/phd/vargaa/omnetpp.htm. 